\title{
Central Auditory Processing Tests as Diagnostic Tools for the Early Identification of Elderly Individuals with Mild Cognitive Impairment
}

\author{
Bahram Jalaei ${ }^{1}$, Ayub Valadbeigi ${ }^{1}$, Rasool Panahi ${ }^{1}$, Morteza Hamidi Nahrani ${ }^{1}$, \\ Hossein Namvar Arefi', Maryam Zia ${ }^{2}$, and Nastaran Ranjbar ${ }^{3}$ \\ ${ }^{1}$ Department of Audiology, School of Rehabilitation Sciences, Iran University of Medical Sciences, Tehran, Iran \\ ${ }^{2}$ Department of Audiology, School of Rehabilitation Sciences, Tehran University of Medical Sciences, Tehran, Iran \\ ${ }^{3}$ Department of Neuroscience, Faculty of Advanced Technologies in Medicine, Iran University of Medical Sciences, Tehran, Iran
}

Received June 14, 2018

Revised September 2, 2018

Accepted October 3, 2018

\section{Address for correspondence Ayub Valadbeigi, MS \\ Department of Audiology, \\ School of Rehabilitation Sciences, Iran University of Medical Sciences, Nezam St., Shah-Nazari St., Madar Sq., Mirdamad Blvd., Tehran, Iran \\ Tel $+98-021-22250541$ \\ Fax +98-021-22227124 \\ E-mail Valadbeigi63@gmail.com}

Background and Objectives: Mild cognitive impairment (MCl) is a disorder that usually occurs in the elderly, leading to dementia in some progressive cases. The purpose of this study is to examine the utility of central auditory processing tests as early diagnostic tools for identifying the elderly with $\mathrm{MCl}$. Subjects and Methods: This study was conducted on 20 elderly patients with $\mathrm{MCl}$ and 20 healthy matched peers. The speech perception ability in a quiet environment and in the presence of background noise and also temporal resolution were assessed by using Speech Perception in Noise (SPIN) and Gap in Noise (GIN) tests, respectively. Results: The results indicated that the ability to understand speech in a quiet environment did not differ significantly between the two groups. However, SPIN at the three signal-tonoise ratios and the temporal resolution scores were significantly different between the two groups $(p<0.001)$. Conclusions: Individuals with $\mathrm{MCl}$ appear to have poorer speech comprehension in noise and a lower temporal resolution than those of the same age, but without cognitive defects. Considering the utility of these tests in identifying cognitive problems, we propose that since the GIN test seems to be less influenced by intervening factors, this test can therefore, be a useful tool for the early screening of elderly people with cognitive problems.

J Audiol Otol 2019;23(2):83-88

KEY WORDS: Dementia · Mild cognitive impairment $\cdot$ Speech perception Auditory processing disorder $\cdot$ Cognition.

\section{Introduction}

Improvements in health care over the past 50 years have led to an increase in life expectancy, especially in people older than 65 years. The number of people who are getting older is increasing and this can increase the prevalence of age-related disorders, such as dementia [1]. Among the normal population, mental abilities may decrease as a result of aging, but the trend is different for people with dementia [2]. Mild cognitive impairment (MCI) may occur in the elderly as a transi-

This is an Open Access article distributed under the terms of the Creative Commons Attribution Non-Commercial License (https://creativecommons.org/licenses/by-nc/4.0/) which permits unrestricted non-commercial use, distribution, and reproduction in any medium, provided the original work is properly cited. tory phase between the normal decline in neurological processes associated with aging and Alzheimer's disease, even though not all MCI cases end in Alzheimer's disease [3,4]. The different definitions of the disorder can be generally divided into two categories: one category includes the definitions that focus on memory impairment and the other pertains to those that include defects in other cognitive fields. However, Graham, et al. [5] presented one of the most comprehensive definitions by describing it as memory, learning, perceptualmotor and linguistic deficiencies in the absence of dementia. Previous studies have provided evidence of impairment in central auditory processing in individuals with MCI. It has been shown that their hearing performance is lower than expected, especially in the presence of background noise or 
competitive noise [6]. According to previous studies, it seems that central auditory processing disorders (CAPDs) are more common in older people who are more likely to develop cognitive problems, including MCI and Alzheimer's disease [7]. It is necessary to consider the hearing status when evaluating MCI patients because it has been shown that there is a relationship between hearing problems and cognitive functions [8]. The diagnosis of MCI in the elderly may be due to hearing impairment, which is common in this group, rather than a real disorder in cognitive functioning. Accordingly, the use of auditory tests can help in identifying patients with MCI and also prevent overestimation of the disease [9]. One of the important points is that though the pure tone audiometry test is commonly used to assess hearing, this evaluation does not assesses central auditory processing. Therefore, it seems that the study of central processing capabilities such as word comprehension in noise and temporal processing in these individuals will show more realistic results for auditory and associated defects. However, since both attention and cognition can affect auditory processing, the differentiation between these two issues can be considered as a different therapeutic process [10]. The auditory and the cognitive processes seem to be interdependent and each one can affect the other's functioning. It has been hypothesized that in CAPD patients, due to the decrease in auditory inputs, more parts of the neural networks that normally participate in memory and attention are involved in cognitive processing and this process can turn into a faulty cycle in which each of these factors exacerbates the others [11]. Since some of the hearing-related processes are affected earlier than the cognitive processes in individuals with cognitive impairments such as MCI, the early detection of cognitive defects may be possible by evaluating the subtle aspects of central auditory processing [12]. The purpose of this study is to examine the possibility of using central auditory processing tests as early diagnostic tools for identifying individuals with MCI.

\section{Subjects and Methods}

\section{Participants}

This study was performed on 20 elderly subjects (13 males, 7 females) aged 65 to 75 years [mean 70.75 years, standard deviation $(\mathrm{SD})=5.09]$ diagnosed with $\mathrm{MCI}$ and 20 matched elderly subjects (12 males, 8 females) without cognitive defects in same age group (mean 71.3 years, $\mathrm{SD}=4.41$ ). The individuals with MCI were selected from a specialized neurological clinic based on their medical records. The Persian version of the Mini-Mental Status Exam (MMSE) questionnaire was used to evaluate the cognitive status of the control group sub- jects [13]. In order to ensure the absence of pathology of the external and middle ear, the subjects underwent otoscopy examination, tympanometry, and audiometric evaluation. The inclusion criteria for all the subjects were: normal hearing thresholds (air conduction thresholds of less than or equal to $25 \mathrm{~dB} \mathrm{HL}$ at octave frequencies of $250-4,000 \mathrm{~Hz}$ ), righthandedness, native Persian language speaker, and without history of epilepsy, seizures, use of psychosocial drugs, head trauma, and ear diseases. All the subjects had a diploma or higher academic degree. In the MCI group, in addition to having the above criteria, it was mandatory for the subjects to have a MCI, according to an expert and the MMSE evaluation result. Written informed consent was obtained from all the subjects. This study was approved by the Ethics Committee of Iran University of Medical Sciences, (IR.IUMS.REC 1396.922 1303201), in accordance with the 1975 Declaration of Helsinki and its later amendments.

\section{Stimuli}

After ensuring normal hearing and middle ear functioning, central auditory processing was evaluated by using the Speech Perception in Noise (SPIN) and Gap in Noise (GIN) tests. The evaluations were carried out in an acoustic booth by using a two-channel Primus audiometer (Auditdata, Copenhagen, Denmark).

\section{SPIN test}

This test consists of four lists of 50 single-syllabic words, which are presented in four different signal-to-noise ratios (SNRs): -20, -10, 0 and $+10 \mathrm{~dB}$. The speech perception ability was evaluated as a function of the SNR in the presence of continuous broadband noise. Each subject was instructed to listen to the presented words and write them on a sheet [14]. It should be noted that the use of all the four lists is not obligatory, and according to the structure and purpose of the study, the desired SNR can be applied. In the present study, with regard to the difficulty of testing at the $-20 \mathrm{~dB}$ SNR, the test was not performed at this level [15]. The test materials were recorded on a CD and presented via an audiometer, while the level of output intensity and test ear were adjusted. The SPIN test was performed at a comfortable hearing level at $40 \mathrm{~dB}$ sensation level. Higher SNRs were used in order to familiarize the subjects with the test procedure. The words in different lists were randomly presented with the mentioned SNRs.

\section{GIN test}

This test composed of four different lists that contained a series of up to 36 different 6-second white noise segments. Each white noise segment contained anywhere from zero to 
three gaps of silence, and the duration of these gaps included $2,3,4,5,6,8,10,12,15$ and $20 \mathrm{~ms}$, with each silence gap duration being represented six times in each GIN list. Therefore, there were 60 gaps per list and also a gap of five seconds of silence in which each noise segment was separated. A practice list to ensure that the patients understood the task was performed. The test was performed monaurally and initiated randomly on either the right or the left ear of each patient. The subjects were asked to respond by pressing a button when they heard the silence gap. If the subject pressed the button when there was no gap, it was considered as a false positive, and when there was a gap, but the button was not pressed, it was considered as an error response. If the subject was confused, he/she was asked to count the number of gaps. There are two criteria for rating the test results: the approximate threshold and the percentage of correct answers. The approximate threshold is the smallest gap that the subject detects correctly in at least four out of the six presentations. The percentage of correct answers is defined as the total number of correct answers for all the gap durations in each list for each ear. Two false positives were negligible and, if there were more, the way to respond was explained to the subject. According to the developer's opinion, two lists are sufficient to perform the GIN test; therefore, two lists were used to avoid subject fatigue and reduce test time as well. We used the GIN test originally developed by Musiek, et al. $[16,17]$.

\section{Statistical analysis}

The test results were analyzed by Statistical Package for Social Sciences [SPSS software ver.16 (SPSS Inc., Chicaco, IL, USA)]. Descriptive statistics were presented as the mean and the SD. The Kolmogorov-Smirnov test was performed to determine if the sample data had a normal distribution. Analytic statistics were done by using the independent samples T-test for inter-group comparisons. A $p$-value of $<0.05$ was determined to be statistically significant.

\section{Results}

\section{SPIN test}

Table 1 and 2 show the mean and the SD of SPIN scores for the right and left ears at the different SNRs used in this study. Significant statistical differences were obtained in the right ear between the two groups for all the three SNRs of $+10,0$ and $-10 \mathrm{~dB}(p<0.001)$. In the absence of background noise, there was no significant difference in the speech perception scores between the two groups $(p=0.650$ ). The same results were obtained for the left ear so that there was a significant statistical difference between all the three SNRs $(p<$
Table 1. Mean and SD of word recognition scores under noisy and quiet conditions for the right ear

\begin{tabular}{|c|c|c|c|c|c|c|}
\hline \multicolumn{2}{|c|}{ Group } & \multicolumn{2}{|c|}{ Normal } & \multicolumn{2}{|c|}{$\mathrm{MCl}$} & \multirow{2}{*}{$P$} \\
\hline Condition & SNR (dB) & Mean & SD & Mean & SD & \\
\hline \multirow[t]{3}{*}{ In noise } & +10 & 74.3 & 3.3 & 66.9 & 4.6 & $<0.001$ \\
\hline & 0 & 59.8 & 4.1 & 53.7 & 4.1 & $<0.001$ \\
\hline & -10 & 24.5 & 3.6 & 20.7 & 2.9 & $<0.001$ \\
\hline In quiet & - & 84.2 & 4.2 & 83.6 & 3.9 & 0.650 \\
\hline
\end{tabular}

SD: standard deviation, SNR: signal-to-noise ratio, $\mathrm{MCl}$ : mild cognitive impairment

Table 2. Mean and SD of word recognition scores under noisy and quiet conditions for the left ear

\begin{tabular}{|c|c|c|c|c|c|c|}
\hline \multicolumn{2}{|c|}{ Group } & \multicolumn{2}{|c|}{ Normal } & \multicolumn{2}{|c|}{$\mathrm{MCl}$} & \multirow{2}{*}{$p$} \\
\hline Condition & SNR (dB) & Mean & $S D$ & Mean & $S D$ & \\
\hline \multirow[t]{3}{*}{ In noise } & +10 & 73.9 & 3.8 & 67.4 & 3.2 & $<0.001$ \\
\hline & 0 & 59.8 & 4.4 & 54.8 & 4.0 & $<0.001$ \\
\hline & -10 & 25.7 & 3.9 & 19.1 & 3.7 & $<0.001$ \\
\hline In quiet & - & 83.3 & 3.4 & 82.3 & 3.2 & 0.393 \\
\hline
\end{tabular}

SD: standard deviation, SNR: signal-to-noise ratio, $\mathrm{MCl}$ : mild cognitive impairment

0.001), but no significant difference in the speech perception scores in the absence of background noise $(p=0.393)$.

\section{GIN test}

The MCI group showed significantly poorer results than the control group in the GIN test. The mean of the approximate threshold of gap detection in the right ear in the control group was $8.7 \mathrm{~ms}( \pm 2.12 \mathrm{SD})$ and in the MCI group, it was $10.8 \mathrm{~ms}( \pm 1.76 \mathrm{SD})$. The mean of the approximate threshold in the left ear of the control group was $8.8 \mathrm{~ms}( \pm 1.36 \mathrm{SD})$ and in the MCI group, it was $11.4 \mathrm{~ms}( \pm 1.95 \mathrm{SD})$. The mean percentage of correct answers in the right ear in the control group was $51.8 \%( \pm 3.63 \mathrm{SD})$ and in the MCI group, it was $40.8 \%$ ( $\pm 4.12 \mathrm{SD})$. For the left ear, the mean percentage of correct answers in the control group and the MCI group was $50.85 \%( \pm 4.12 \mathrm{SD})$ and $40.01 \%( \pm 4.18 \mathrm{SD})$, respectively. The analysis of the GIN results, including the approximate thresholds and the correct responses between the right and the left ears, of the two groups showed a significant difference $(p<0.001)$. The comparison between the two groups is illustrated in Fig. 1 and 2.

\section{Discussion}

\section{Speech perception in quiet}

Hearing loss is generally considered as an increase in average pure tone thresholds. But hearing ability is beyond pure tone sensitivity and includes more factors such as frequency selectivity, temporal coding fidelity, temporal encoding, inten- 


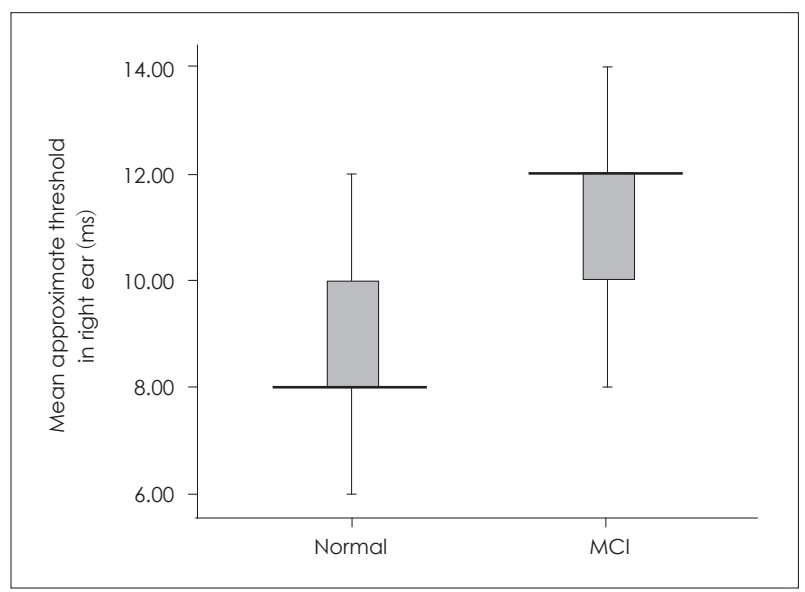

Fig. 1. Mean and SD of the approximate threshold of GIN test of normal and $\mathrm{MCl}$ subjects in the right ear. SD: standard deviation, GIN: gap in noise, $\mathrm{MCl}$ : mild cognitive impairment.

sity resolution, loudness, etc., which are not normally measured during an audiometric evaluation [6]. As we expected, the results of this study showed that speech perception in quiet did not differ significantly between the two groups. This conclusion confirms that if there is no competitive noise, the hearing ability of the elderly will not be affected considerably. Many elderly people usually complain about a difficulty to understand speech in the presence of background noise even though they have a normal hearing threshold. Therefore, it can be said that speech comprehension in noise depends on both hearing condition and cognitive functioning $[18,19]$. Sole focus on pure tone measurements results in an underestimation of cognitive performance. It has been shown that changes in hearing thresholds and the rate of hearing loss progression are higher in people with a probable cognitive impairment [20]. By carefully monitoring the interfering factors, it has been shown that hearing loss of more than $25 \mathrm{~dB}$ can cause or intensify cognitive impairment [21]. It is estimated that the rate of hearing loss in the elderly with dementia is almost twice as high as in their peers without dementia. On the other hand, some studies have reported no significant relationship between hearing loss and cognition [22]. Accordingly, in order to reduce the impact of peripheral hearing loss on the results of this study, individuals with normal peripheral hearing were included. The subjects with some degree of cognitive problems seemed to have difficulty in processing the auditory inputs efficiently. For such situations, in which the acoustic environment is inappropriate or speech stimuli is accompanied by noise, taking advantage of the cognitive abilities associated with auditory processing is necessary in order to properly understand the message and correctly process the auditory signal. For these reasons, individuals who have cognitive problems and cannot benefit from cognitive abilities,

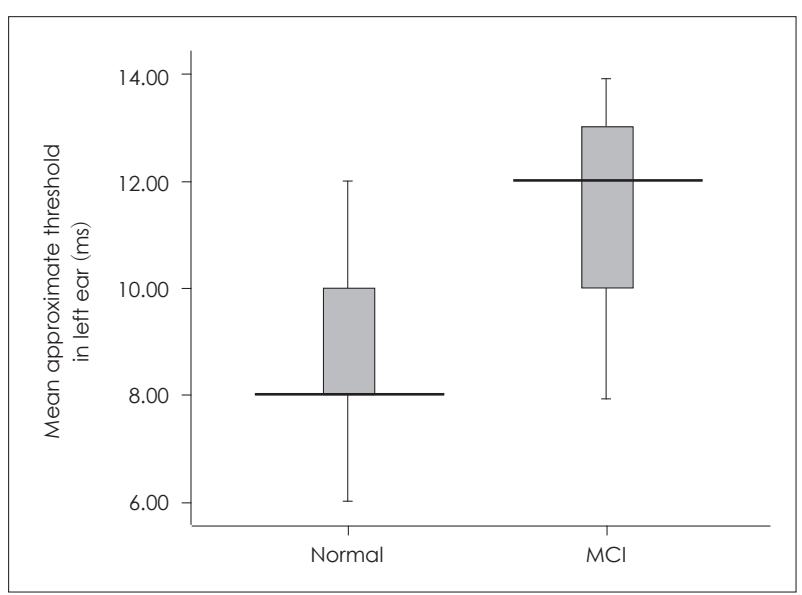

Fig. 2. Mean and SD of the approximate threshold of GIN test of normal and $\mathrm{MCl}$ subjects in the left ear. SD: standard deviation, GIN: gap in noise, $\mathrm{MCl}$ : mild cognitive impairment.

experience difficulties in speech comprehension in such demanding situations. The results of this study are in line with previous investigations [23].

\section{Speech perception in noise}

Age-related changes in cognitive functions can be a result of the changes in general sensory processing among the elderly [24]. The use of simple clinical measures to investigate sensory processing is not enough to detect the sensory impairment associated with cognitive impairment. Also, for a better understanding of speech consonants, advanced hearing skills such as temporal processing and prosody are needed. Therefore, for individuals with CAPD, speech perception becomes difficult, especially in noisy and inappropriate acoustic environments. The results of the present study showed that the ability of word perception in the presence of noise in the MCI group was significantly poorer than the control group; this finding was in line with the results of previous studies [25]. The connection between hearing impairment and cognitive impairment is inevitable; however, if only the pure tone threshold is used, this relationship will be weak and will be less than expected. Central auditory processing impairment can play a role in the development of more advanced cognitive diseases. It can reduce communication between an elderly person and associates and isolate him/her [12]. This isolation may lead to serious illnesses such as Alzheimer's diseases. A long-term cohort study has shown this relationship [7]. The results for speech perception in noise in elderly MCI subjects were significantly lower than the elderly control subjects. Although these findings do not explain the origin of the problem certainly and whether these CAPDs caused this difference or cognitive problems, what was clear is that there was difficulty in understanding speech in the presence of noise in the MCI 
group. For this reason, the tools used in this study were selected in such a way that we could differentiate between the cognitive and auditory problems to some extent. Another important finding of this study was that the reduction of peripheral hearing sensitivity did not interfere with the performance of individuals in a noisy environment. This is because despite normal hearing sensitivity in many individuals with CAPD, they still have a difficulty in challenging hearing situations [26]. It has been reported that central auditory tests, rather than cognitive screening tests, are more sensitive in identifying dysfunctions caused by neurodegeneration of the cortical regions. Therefore, the assessment of central auditory processing may be an appropriate screening tool and it helps in the early diagnosis of cognitive problems. Previous research has shown that nearly $46 \%$ of older people with CAPD develop Alzheimer's disease within the next five to seven years. It should be noted that people susceptible to Alzheimer's disease have more difficulty in understanding speech in the presence of competitive noise, and that it can be identified earlier [11]. Therefore, this finding suggested that the early detection of CAPDs and the treatment of these disorders can help in improving the communication problems of people with dementia.

\section{Auditory temporal resolution}

The gap detection ability cannot be predicted by an audiogram, and individuals with a similar degree of hearing impairment and audiogram pattern often have a different ability to detect the intervals. Elderly people have a significantly poorer gap detection threshold than young individuals [27,28]. However, its relation with cognitive impairment has not been fully understood. Gap detection tests provide valuable information regarding the ability of individuals suspected to have CAPD [29]. We found that there was a considerable difference between the two groups in the results of the SPIN and GIN tests for both the right and the left ears. So, it seems that due to the effect of cognitive functions on central hearing processing in MCI individuals, their central auditory processing capabilities are more affected than their normal peers. This is perhaps one of the possible reasons for the reduction of MCI performance in auditory temporal processing as compared to individuals of the same age who had no cognitive problems. It is necessary to note that the assessment of speech in noise, including the SPIN test, causes more cognitive load, and is influenced by more interfering factors [30]. However, the GIN test evaluates rapid changes in the auditory stimuli; although it is affected by advancing age, it less affected by hearing loss and so it is a better tool for utilization among elderly people.

\section{Conclusion}

The results of this study showed that elderly people with MCI have a significantly lower ability to understand speech signals in the presence of background noise as compared to those of the same age, but without cognitive problems. They also have a lower ability for auditory temporal resolution. We suggest the evaluation of central auditory processing abilities as a clinical tool in identifying elderly people with cognitive problems. Given that the GIN test is less influenced by interfering factors, this test can be considered in the test battery for early screening and help in the identification of elderly individuals with some degree of cognitive problems.

\section{Acknowledgments}

We thank Ava Gostar Sobhan Company for providing the equipment used in this study. We also thank all the participants.

\section{Conflicts of interest}

The authors have no financial conflicts of interest.

\section{REFERENCES}

1) Hebert LE, Beckett LA, Scherr PA, Evans DA. Annual incidence of Alzheimer disease in the United States projected to the years 2000 through 2050. Alzheimer Dis Assoc Disord 2001;15:169-73.

2) Wilson RS, Beckett LA, Bennett DA, Albert MS, Evans DA. Change in cognitive function in older persons from a community population: relation to age and Alzheimer disease. Arch Neurol 1999;56:1274-9.

3) Bozoki A, Giordani B, Heidebrink JL, Berent S, Foster NL. Mild cognitive impairments predict dementia in nondemented elderly patients with memory loss. Arch Neurol 2001;58:411-6.

4) Petersen RC, Stevens JC, Ganguli M, Tangalos EG, Cummings JL, DeKosky ST. Practice parameter: early detection of dementia: mild cognitive impairment (an evidence-based review). Report of the Quality Standards Subcommittee of the American Academy of Neurology. Neurology 2001;56:1133-42.

5) Graham JE, Rockwood K, Beattie BL, McDowell I, Eastwood R, Gauthier S. Standardization of the diagnosis of dementia in the Canadian Study of Health and Aging. Neuroepidemiology 1996;15:246-56.

6) Edwards JD, Lister JJ, Elias MN, Tetlow AM, Sardina AL, Sadeq NA, et al. Auditory processing of older adults with probable mild cognitive impairment. J Speech Lang Hear Res 2017;60:1427-35.

7) Gates GA, Cooper JC Jr, Kannel WB, Miller NJ. Hearing in the elderly: the Framingham cohort, 1983-1985. Part I. Basic audiometric test results. Ear Hear 1990;11:247-56.

8) Baltes PB, Lindenberger U. Emergence of a powerful connection between sensory and cognitive functions across the adult life span: a new window to the study of cognitive aging? Psychol Aging 1997;12: 12-21.

9) Lindenberger U, Baltes PB. Sensory functioning and intelligence in old age: a strong connection. Psychol Aging 1994;9:339-55.

10) Chermak GD, Lee J. Comparison of children's performance on four tests of temporal resolution. J Am Acad Audiol 2005; 16:554-63.

11) Iliadou VV, Bamiou DE, Sidiras C, Moschopoulos NP, Tsolaki M, Nimatoudis I, et al. The use of the Gaps-In-Noise test as an index of the enhanced left temporal cortical thinning associated with the transition between mild cognitive impairment and Alzheimer's disease. J Am Acad Audiol 2017;28:463-71.

12) Gates GA, Anderson ML, McCurry SM, Feeney MP, Larson EB. Central auditory dysfuntion as a harbinger of Alzheimer dementia. Arch Otolaryngol Head Neck Surg 2011;137:390-5. 
13) Foroughan M, Jafari Z, Shirin BP, Ghaem MFZ, Rahgozar M. Validation of Mini-Mental State Examination (MMSE) in the elderly population of Tehran. Adv Cogn Sci 2008;10:29-37.

14) Heidari M, Mahdavi ME, Heidari F, Baghban AA. Auditory recognition of Persian digits in multi-talker babble noise: a preliminary study. Aud Vestib Res 2015;24:134-40.

15) Mahdavi ME, Aghazadeh J, Tahaei SAA, Heiran F, Baghban AA. Persian randomized dichotic digits test: development and dichotic listening performance in young adults. Aud Vestib Res 2017;23:99-113.

16) Musiek FE, Shinn JB, Jirsa R, Bamiou DE, Baran JA, Zaida E. GIN (Gaps-In-Noise) test performance in subjects with confirmed central auditory nervous system involvement. Ear Hear 2005;26:608-18.

17) Valadbeigi A, Weisi F, Rohbakhsh N, Rezaei M, Heidari A, Rasa AR. Central auditory processing and word discrimination in patients with multiple sclerosis. Eur Arch Otorhinolaryngol 2014;271:2891-6.

18) Anstey KJ, Luszcz MA, Sanchez L. A reevaluation of the common factor theory of shared variance among age, sensory function, and cognitive function in older adults. J Gerontol B Psychol Sci Soc Sci 2001;56: 3-11.

19) Northern JL, Downs MP. Hearing in children. Philadelphia: Lippincott Williams \& Wilkins;2002.

20) Wong LL, Yu JK, Chan SS, Tong MC. Screening of cognitive function and hearing impairment in older adults: a preliminary study. Biomed Res Int 2014;2014:867852.

21) Lin FR, Ferrucci L, An Y, Goh JO, Doshi J, Metter EJ, et al. Association of hearing impairment with brain volume changes in older adults. Neuroimage 2014;90:84-92.
22) Lin MY, Gutierrez PR, Stone KL, Yaffe K, Ensrud KE, Fink HA, et al. Vision impairment and combined vision and hearing impairment predict cognitive and functional decline in older women. J Am Geriatr Soc 2004;52:1996-2002.

23) Glasberg BR, Moore BC, Patterson RD, Nimmo-Smith I. Dynamic range and asymmetry of the auditory filter. J Acoust Soc Am 1984;76: 419-27.

24) Humes LE, Busey TA, Craig J, Kewley-Port D. Are age-related changes in cognitive function driven by age-related changes in sensory processing? Atten Percept Psychophys 2013;75:508-24.

25) Idrizbegovic E, Hederstierna $C$, Dahlquist $M$, Kämpfe Nordström $C$, Jelic V, Rosenhall U. Central auditory function in early Alzheimer's disease and in mild cognitive impairment. Age Ageing 2011;40:249-54.

26) Gates GA, Karzon RK, Garcia P, Peterein J, Storandt M, Morris JC, et al. Auditory dysfunction in aging and senile dementia of the Alzheimer's type. Arch Neurol 1995;52:626-34.

27) He NJ, Horwitz AR, Dubno JR, Mills JH. Psychometric functions for gap detection in noise measured from young and aged subjects. $\mathrm{J}$ Acoust Soc Am 1999;106:966-78.

28) Lister JJ, Roberts RA, Lister FL. An adaptive clinical test of temporal resolution: age effects. Int J Audiol 2011;50:367-74.

29) Samelli AG, Schochat E. The gaps-in-noise test: gap detection thresholds in normal-hearing young adults. Int J Audiol 2008;47:238-45.

30) Kiely KM, Gopinath B, Mitchell P, Luszcz M, Anstey KJ. Cognitive, health, and sociodemographic predictors of longitudinal decline in hearing acuity among older adults. J Gerontol A Biol Sci Med Sci 2012;67:997-1003. 